\title{
Expression of neighbor of Punc E11 in hepatocarcinogenesis induced by diethylnitrosamine
}

\author{
YOUNG HEE KIM, KYUNG A KWAK and JIN SEOK KANG \\ Department of Biomedical Laboratory Science, Namseoul University, Cheonan 330-707, Republic of Korea
}

Received March 5, 2014; Accepted April 17, 2014

DOI: $10.3892 /$ or.2014.3285

\begin{abstract}
To identify preneoplastic markers for hepatocarcinogenesis, the expression levels of neighbor of Punc E11 (Nope), E-cadherin and $\alpha$-fetoprotein (AFP) were investigated in carcinogen-treated embryonic cell lineages. Mouse embryonic stem cells (ESCs), hepatic progenitor cells (HPCs), and hepatocyte-like cells (HCs) representing 0, 22, and 40 days of liver differentiation, respectively, were treated in vitro with diethylnitrosamine (DEN) at 4 doses $(0,1,5$, and $15 \mathrm{mM})$ for $24 \mathrm{~h}$. The expression of Nope, E-cadherin and AFP was examined by quantitative real-time PCR, western blotting and immunocytochemistry. DEN treatment significantly increased the mRNA expression of Nope in ESCs and HPCs, and that of E-cadherin and AFP in all three cell lines, although the changes in expression were triggered by varying DEN concentrations. Immunofluorescence staining revealed that Nope was expressed at the cell membrane in ESCs and HPCs and within granules or in the cytoplasm of HCs, which was also stained by E-cadherin. DEN treatment induced Nope expression in ESCs, HPCs and HCs and caused a concomitant increase in E-cadherin expression. Although Nope expression clearly increased following tumor induction, its expression pattern changed with the developmental stage. In conclusion, we determined that Nope expression may carry prognostic significance during early hepatocarcinogenesis. Moreover, Nope expression may serve as a novel oncofetal surface marker for preneoplastic stages that are not identifiable by the commonly used marker, AFP.
\end{abstract}

\section{Introduction}

In toxicology and pharmacology, embryonic stem cell (ESC) derived hepatocytes have been suggested as an in vitro alternative model for toxicity and/or carcinogenicity assay testing $(1,2)$. Hepatic differentiation of mouse ESCs was first

Correspondence to: Professor Jin Seok Kang, Department of Biomedical Laboratory Science, Namseoul University, 91 Daehak-ro, Seonghwan-eup, Sebuk-gu, Cheonan 330-707, Republic of Korea E-mail: kang@nsu.ac.kr

Key words: mouse embryonic stem cells, hepatic lineage cells, hepatocarcinogenesis, neighbor of Punc E11, diethylnitrosamine demonstrated using a stepwise method, adding specific growth factors after the formation of an embryoid body (3). The recent generation of functional hepatocytes from ESCs using chemically defined culture conditions has revealed similarities to in vivo hepatogenesis $(4,5)$. The functionality of differentiated hepatocytes is generally confirmed by the expression of specific marker proteins, such as cytokeratins (CK), GATA binding proteins (GATA), and $\alpha$-fetoprotein (AFP). Expression of these indicators of hepatic development can also be used as a measure of hepatotoxicity.

ESC-derived hepatocytes and hepatic progenitors may also serve as a model to study the induction of hepatocarcinoma, as hepatic tumors originate from liver stem cells (6). Prior to tumor formation, exposure to carcinogens first causes the formation of preneoplastic lesions as an adaptive non-oncogenic response. Similar to numerous solid tumors, hepatocarcinoma is sustained by a distinct subpopulation of cancer stem cells (7).

Hepatocellular carcinoma (HCC) is distinguished from normal liver or dysplastic lesions by the expression of the neighbor of Punc E11 (Nope), a transmembrane protein in the immunoglobulin superfamily. Nope was initially described by Salbaum and Kappen (8) and has high sequence homology with Punc and the axonal guidance receptors, deleted in colorectal cancer and Neogenin (9-11). High levels of Nope expression are noted at the time of transformation from preneoplastic lesion to malignant HCC (12). Moreover, expression levels progressively increase with the advance of HCC, suggesting a potential role of Nope as a prognostic indicator.

Following our observation that diethylnitrosamine (DEN)-induced carcinogenicity in animals varies with age, we hypothesized that DEN-induced expression of Nope would vary with the differentiation stage of hepatic cells. In the present study, we investigated how sensitivity to DEN, as measured by the expression of Nope, varies with hepatic differentiation from ESCs to mature hepatocytes.

\section{Materials and methods}

Culture of mouse ESCs and differentiation of hepatic lineage cells. Mouse ESCs (NVRQS-11F) were cultured using mitomycin C-treated mouse embryonic fibroblasts as feeder cells on $0.1 \%$ gelatin-coated dishes in Dulbecco's modified Eagle's medium (DMEM) (Millipore, Billerica, MA, USA) supplemented with $15 \%$ fetal bovine serum (Invitrogen, 
Table I. Murine oligonucleotide primer sequences used for quantitative RT-PCR.

\begin{tabular}{lccc}
\hline Gene name & Accession number & Primer sequence & Product length (bp) \\
\hline Nope & NM_020043 & 5'-CCTGGTATATGACGCCATAA-3' & 90 \\
AFP & NM_007423 & 5'-GAGTGGACAATGACCTCAG-3' & 75 \\
E-cadherin & NM_009864 & 5'-CCTGTTGGAATACGAAGAGTT-3' & \\
GAPDH & 5M_008084 & 5'-CTGTGGGGGGATGGATGAGAG-3' & 115 \\
& & 5'-GAAACCTGCCAAGTATGATG-3' & 121 \\
\hline
\end{tabular}

Rockville, MD, USA), 2 ml-glutamine (Millipore), $0.1 \%$ nonessential amino acids (Invitrogen), $1 \%$ penicillin-streptomycin (Millipore), and $10 \mathrm{ng} / \mathrm{ml}$ mouse leukemia inhibitory factor (Millipore). To differentiate ESCs into hepatic lineage cells (HPCs) in vitro, defined culture media were supplemented with rmHGF (Invitrogen), dimethyl sulfoxide (DMSO) and sodium butyrate (Sigma-Aldrich, St. Louis, MO, USA). Subsequently, mEGF (Invitrogen), oncostatin M, dexamethasone, nicotinamide and ascorbic acid (Sigma-Aldrich) were added to differentiate HCs from HPCs.

DEN treatment of ESCs, HPCs and HCs. ESCs (day 0), HPCs (day 22 of differentiation) and HCs (day 40 of differentiation) were treated with four concentrations of $\operatorname{DEN}(0,1,5$ and $15 \mathrm{mM}$ ) for $24 \mathrm{~h}$.

Nope and E-cadherin mRNA expression. RNA was isolated from cultured cells using an RNeasy Mini Kit (Qiagen, Valencia, CA, USA), dissolved in diethylpyrocarbonate (DEPC)-treated distilled water and stored at $-80^{\circ} \mathrm{C}$ until use. RNA concentrations were measured spectrophotometrically. Nope and GAPDH mRNA expression was determined by relative quantitative real-time PCR in 96-well optical plates using an ABI StepOnePlus ${ }^{\mathrm{TM}}$ Real-Time PCR System (Applied Biosystems, Foster City, CA, USA). The primers are listed in Table I. The expression levels of the target genes were normalized to mouse GAPDH mRNA and are expressed as the relative expression. Gene expression was normalized according to the cycle number at which the fluorescence signal of the target product was detectable (threshold cycle, $\mathrm{Ct}$ ) to provide $\Delta \mathrm{Ct}$. The expression of the genes relative to a reference was calculated as $2^{-\Delta \Delta \mathrm{Ct}}$, where $\Delta \Delta \mathrm{Ct}$ refers to the difference between the $\Delta \mathrm{Ct}$ values of the test group and the reference.

Immunofluorescence staining of Nope and E-cadherin. Cells were cultured at a low density on coverslips and fixed in $4 \%$ paraformaldehyde for $15 \mathrm{~min}$ at room temperature (RT). The medium was removed by aspiration, and cells were washed twice with $1 \mathrm{X}$ phosphate-buffered saline (PBS; pH 7.4; Invitrogen), fixed in $4 \%$ paraformaldehyde (Sigma-Aldrich) for $5 \mathrm{~min}$, and rinsed twice with $1 \mathrm{X}$ PBS. After cell permeabilization in $1 \mathrm{X}$ PBS and $0.1 \%$ Triton X-100 (PT) for $10 \mathrm{~min}$, proteins were blocked with $4 \%$ normal goat serum in PT and incubated with anti-Nope (1:100 dilution; R\&D Systems, Minneapolis, MN,

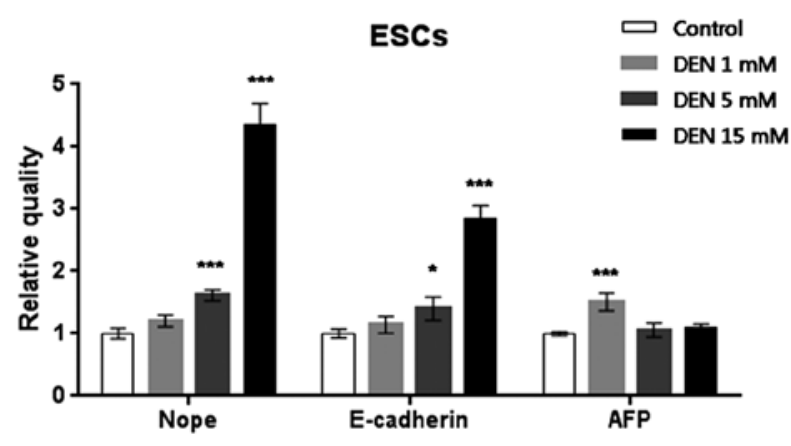

Figure 1. Tumor marker expression in DEN-treated mouse embryonic stem cells (ESCs). Expression levels of neighbor of Punc E11 (Nope), E-cadherin and $\alpha$-fetoprotein (AFP) were analyzed by quantitative RT-PCR in mouse embryonic stem cells (ESCs) treated with diethylnitrosamine (DEN) at four doses $(0,1,5$ and $15 \mathrm{mM})$ for $24 \mathrm{~h} .{ }^{* *} \mathrm{P}<0.01,{ }^{* * *} \mathrm{P}<0.001 ; \mathrm{n}=12$ from three independent experiments.

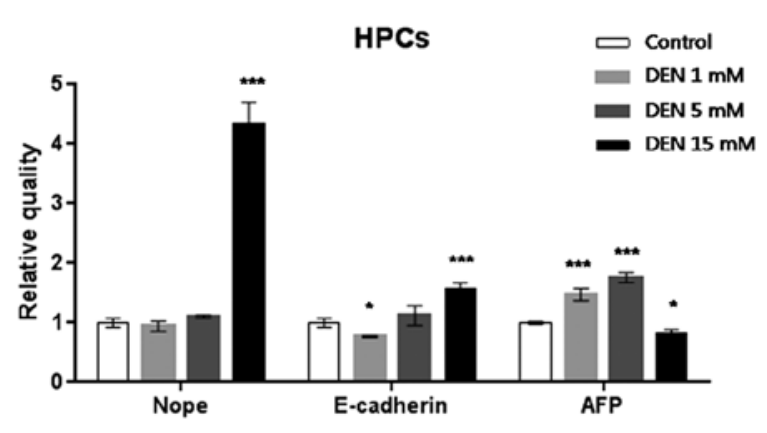

Figure 2. Tumor marker expression in DEN-treated hepatic progenitor cells (HPCs). Expression levels of neighbor of Punc E11 (Nope), E-cadherin and $\alpha$-fetoprotein (AFP) were analyzed by quantitative RT-PCR in hepatic progenitor cells from mouse embryonic stem cells treated with diethylnitrosamine (DEN) at four doses $(0,1,5$ and $15 \mathrm{mM})$ for $24 \mathrm{~h} .{ }^{* *} \mathrm{P}<0.01$, ${ }^{* * * *} \mathrm{P}<0.001 ; \mathrm{n}=12$ from three independent experiments.

USA). Hepatic lineage cells were incubated in anti-E-cadherin (1:100 dilution; Abcam, Cambridge, MA, USA) for $1 \mathrm{~h}$ at RT. Cells were then incubated with Alexa488-conjugated antirabbit IgG and Alexa597-conjugated anti-mouse IgG/IgM (Invitrogen) for $1 \mathrm{~h}$ at RT and counterstained with Hoechst 33258. After three washes with PBS, cells were mounted in Fluorescence Mounting Medium (Dako, Glostrup, Denmark). Immunofluorescence was detected under a confocal microscope (LSM-700; Carl Zeiss, Thornwood, NY, USA). 


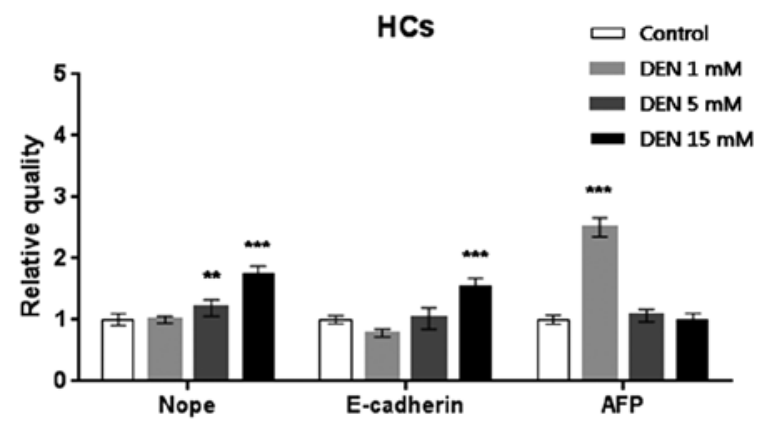

Figure 3. Tumor marker expression in DEN-treated hepatic like cells (HCs). Expression levels of neighbor of Punc E11 (Nope), E-cadherin and $\alpha$-fetoprotein (AFP) were analyzed by quantitative RT-PCR in hepatic like cells from mouse embryonic stem cells treated with diethylnitrosamine (DEN) at four doses $(0,1,5$ and $15 \mathrm{mM})$ for $24 \mathrm{~h} .{ }^{* *} \mathrm{P}<0.01,{ }^{* * *} \mathrm{P}<0.001 ; \mathrm{n}=12$ from three independent experiments.

Statistical analysis. Statistical analyses were performed using GraphPad Prism 5 (GraphPad Software, La Jolla, CA, USA). All data were analyzed using the Mann-Whitney U test and are expressed as the mean $\pm \mathrm{SD}$ of at least three independent experi- ments performed in triplicate or quadruplicate. P-values $<0.05$ were considered to indicate statistically significant results.

\section{Results}

Nope, E-cadherin and AFP mRNA expression. ESCs treated with 5 or $15 \mathrm{mM}$ DEN expressed 1.5- or 4.5-fold greater levels of Nope, respectively, when compared with normal ESCs $(\mathrm{P}<0.001)$. Expression level of E-cadherin increased significantly by 3 - and 1.3 -fold in the ESCs treated with 5 or $15 \mathrm{mM}$ DEN ( $\mathrm{P}<0.05$ and $\mathrm{P}<0.001$, respectively). AFP mRNA expression increased significantly 1.5 -fold in the ESCs treated with $1 \mathrm{mM}$ DEN $(\mathrm{P}<0.001)$, as compared to the control ESCs (Fig. 1).

HPCs treated with $15 \mathrm{mM}$ DEN expressed 4.5-fold higher levels of Nope expression compared with the normal ESCs $(\mathrm{P}<0.001)$. Expression of E-cadherin significantly increased 1.5 -fold in the ESCs treated with DEN $15 \mathrm{mM}(\mathrm{P}<0.001)$. AFP mRNA expression increased significantly 1.5 - and 2 -fold in the HPCs treated with 1 and $5 \mathrm{mM}$ DEN $(\mathrm{P}<0.001)$, respectively, relative to the control HPCs (Fig. 2).

HCs treated with 5 or $15 \mathrm{mM}$ DEN expressed 1.2- or 1.5 -fold higher levels of Nope relative to the untreated HCs

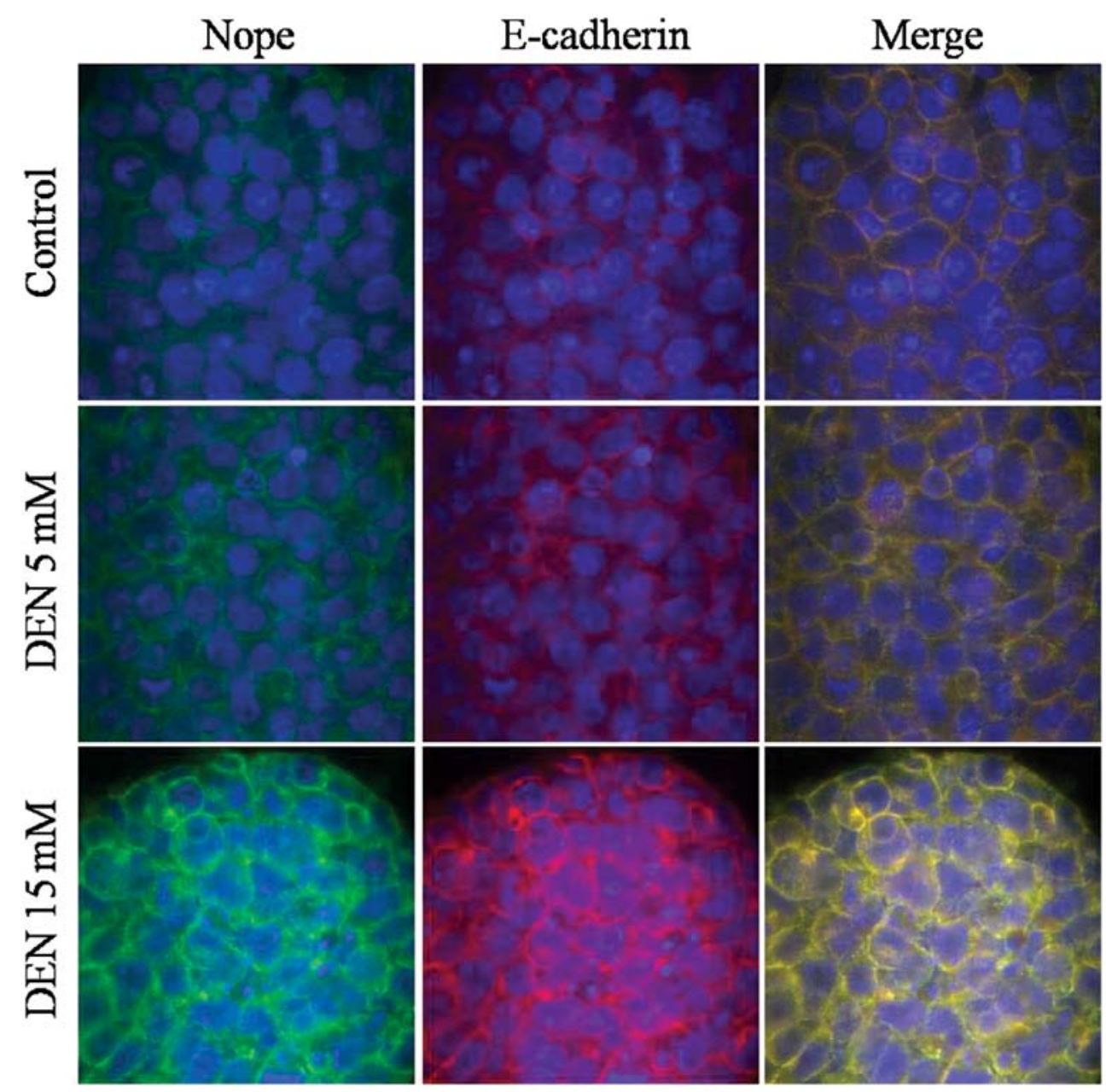

Figure 4. Immunocytochemistry of neighbor of Punc E11 (Nope) and E-cadherin during the development of the liver in embryonic stem cells (ESCs) modulated by diethylnitrosamine (DEN) treatment and confocal microscopy for detection of Nope in ESCs was carried out. Immunofluorescence stainings for Nope (green) showed a membranous and/or cytoplasm expression pattern. Epithelial cells were defined by costaining for E-cadherin (red), proving that all Nope-positive cells were of epithelial origin (merged image). Nuclei were stained with 4',6-diamidino-2-phenylindole (DAPI) (blue). 


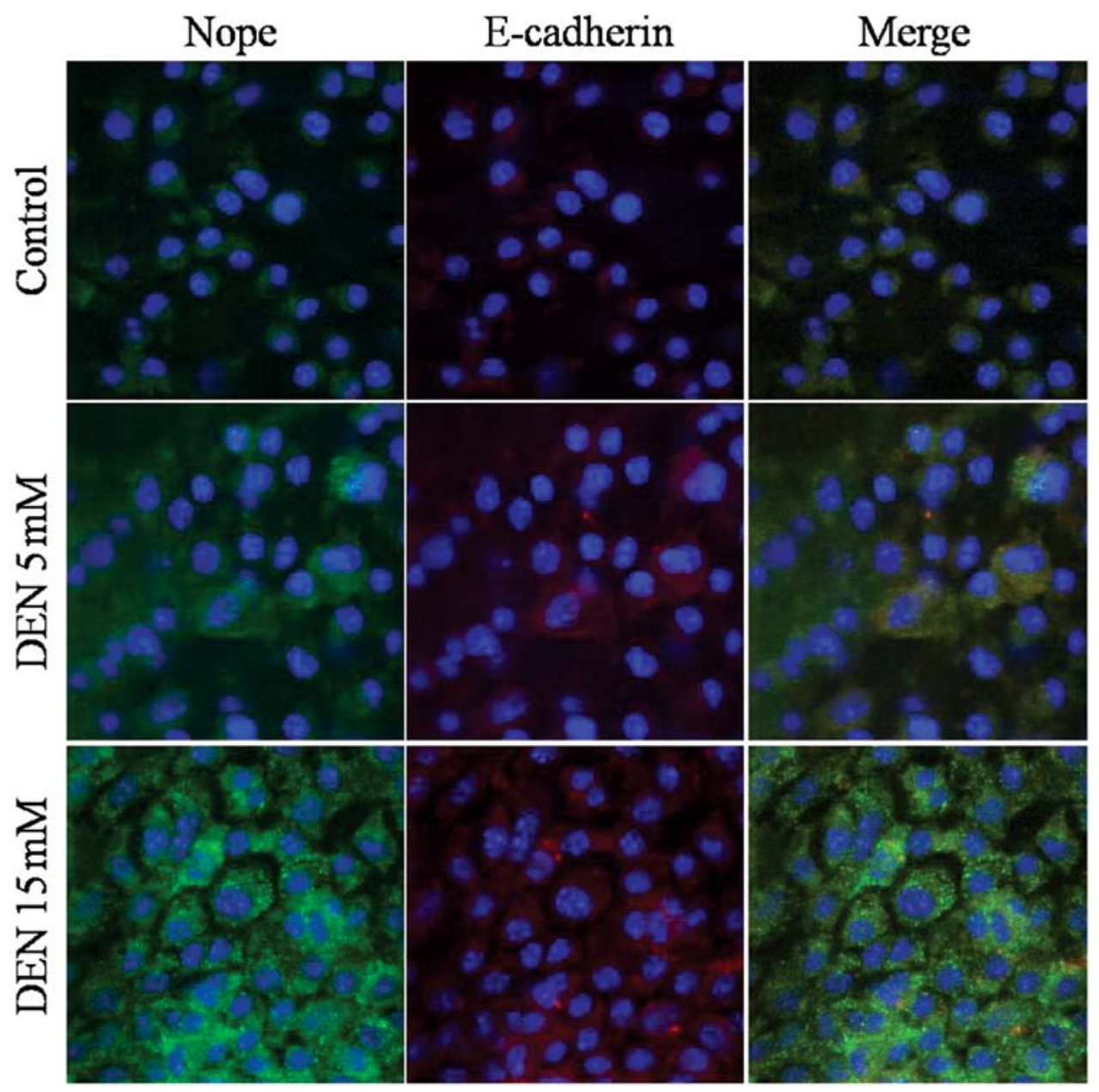

Figure 5. Immunocytochemistry of neighbor of Punc E11 (Nope) and E-cadherin during the development of the liver, in hepatic progenitor cells (HPCs) modulated by diethylnitrosamine (DEN) treatment, and confocal microscopy for detection of Nope in HPCs was carried out. Immunofluorescence stainings for Nope (green) showed a membranous and/or cytoplasm expression pattern. Epithelial cells were defined by costaining for E-cadherin (red), proving that all Nope-positive cells were of epithelial origin (merged image). Nuclei were stained with 4',6-diamidino-2-phenylindole (DAPI) (blue).

$(\mathrm{P}<0.01, \mathrm{P}<0.001$, respectively). Expression of E-cadherin increased by 3 - and 1.3 -fold in the HCs treated with 5 and $15 \mathrm{mM}$ DEN, respectively $(\mathrm{P}<0.001)$. AFP mRNA expression increased significantly by 2.2 -fold in the HCs treated with $1 \mathrm{mM}$ DEN $(\mathrm{P}<0.001)$ as compared to the control HCs (Fig. 3).

Expression of Nope and E-cadherin increased in a dosedependent manner with the concentration of DEN in the cultured hepatic lineage cells. However, AFP mRNA expression increased only at lower concentrations of DEN (1 or $5 \mathrm{mM}$ ).

Immunofluorescence staining of Nope and E-cadherin. Immunofluorescence staining for Nope revealed membranous expression in hepatic lineage cells derived from mouse ESCs, as well as the co-expression of E-cadherin. Merged images indicate that all cells were positive for both markers.

The cellular distribution of Nope was also compared to that of E-cadherin induced by exposure to DEN. Nope was specifically detected at the cell surface in cells treated with high doses of DEN in the ESCs and HPCs.

DEN treatment increased Nope and E-cadherin expression in ESCs, HPCs and HCs (Figs. 4-6). Furthermore, in ESCs treated with DEN, Nope expression not only increased but followed the same cell membrane pattern as E-cadherin (Fig. 4). During differentiation into HPCs and HCs, Nope and E-cadherin expression decreased, while DEN treatment reversed this trend (Figs. 5 and 6).

\section{Discussion}

Given that no effective treatment exists for HCC, and upon diagnosis, most patients with advanced disease have a remaining life-span of 4-6 months, the early detection of HCC is crucial (13). Serum AFP concentrations are often elevated in patients with HCCs, but can only be detected at advanced stages (14).

AFP, first described by Abelev et al in 1960, is the most intensively investigated tumor marker for HCC. AFP is an oncofetal glycoprotein with a molecular weight of $70 \mathrm{kDa}$ and unknown function (15). It is physiologically expressed in fetal liver and pathologically increased in the serum of many patients with $\mathrm{HCC}$, but its sensitivity and specificity are limited (60-70 and 80-90\%, respectively) (16,17). For many years, AFP was the only marker available to identify HCC development in patients at high risk. The combination with ultrasound improves the detection rate of AFP, but a high percentage of 


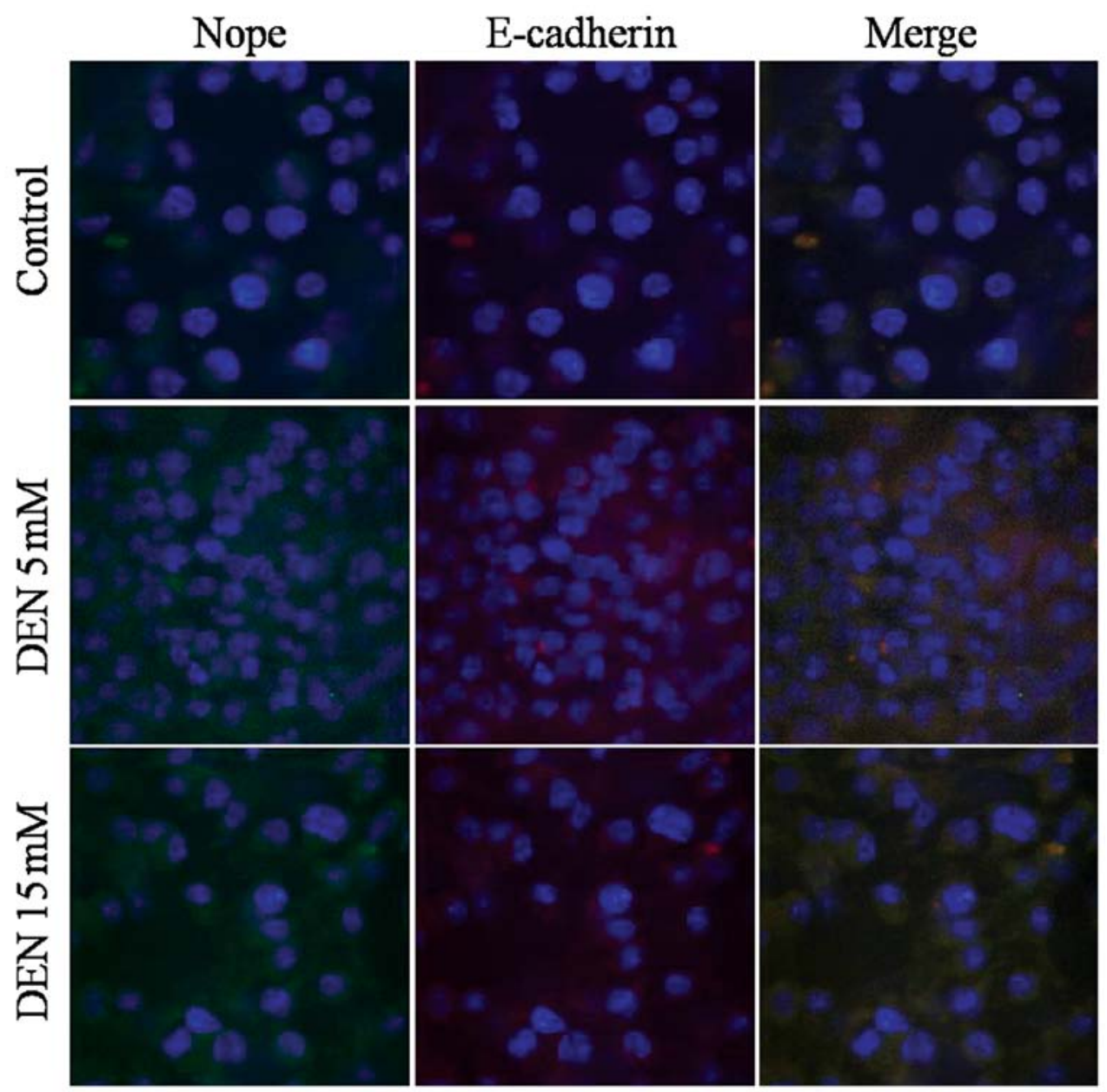

Figure 6. Immunocytochemistry of neighbor of Punc E11 (Nope) and E-cadherin during the development of the liver, in hepatocyte-like cells (HCs) was modulated by diethylnitrosamine (DEN) treatment and confocal microscopy for detection of Nope in HCs was carried out. Immunofluorescence stainings for Nope (green) showed a membranous and/or cytoplasm expression pattern. Epithelial cells were defined by costaining for E-cadherin (red), proving that all Nope-positive cells were of epithelial origin (merged image). Nuclei were stained with 4',6-diamidino-2-phenylindole (DAPI) (blue).

patients with HCC do not present with elevated AFP levels and vice versa. Although recent clinical trials indicate that AFP is more sensitive in detecting HCC than other newly identified markers, current guidelines no longer recommend AFP for surveillance due to its unreliability (11).

Marquardt et al reported the potential of Nope as an oncofetal surface marker for murine and human HCC, and provided evidence for its specific expression in hepatoma cell lines and primary HCC. Nope expression was selectively detected in HCC specimens but not in normal liver or dysplastic lesions, and increases in Nope expression coincided with the transformation of preneoplastic lesions to malignant HCC. Nope was found to be expressed at higher levels than AFP, and was $100 \%$ sensitive and specific for HCC in the selected mouse model (12).

In the present study, expression of Nope was independent of AFP expression in the DEN-treated hepatic lineage cells from mouse ESCs. Although Nope expression increased with greater concentrations of the carcinogen, AFP expression remained low following treatment with lower concentrations of the carcinogen. Nope was expressed at higher levels than AFP in the hepatic lineage cells derived from mouse ESCs. Several studies have not been able to detect increased AFP in dysplastic lesions and early HCC; AFP expression appears to be specifically expressed by advanced HCC (18-20). Thus, promising markers for the specific detection of preneoplastic and early $\mathrm{HCC}$ are needed. Nope seems to be a promising marker for specific, early detection of HCC that could complement other markers.

Although initial studies to identify and enrich for hepatoblasts or endodermal cells in the fetal liver relied on combinations of known stem cell surface markers expressed by many different cell types within the liver $(21,22)$, more recent studies have shown that Liv2 (23), $\delta$-like 1 homolog (Dlk1) (Drosophila) (24), and E-cadherin $(25,26)$ are specific cell surface markers of fetal liver stem/progenitor cells (FLSPCs) within the developing liver.

In the present study, we detected high expression levels of Nope in DEN-treated hepatic lineage cells. DEN, a wellknown hepatocarcinogen, is widely used in mouse liver cancer models (27-32). Expression of E-cadherin and AFP was also highly elevated in DEN-treated cells as compared to untreated cells. When the concentration of DEN was varied $(1,5$ and $15 \mathrm{mM}$ ), the mRNA expression of Nope and E-cadherin increased in a dose-dependent manner. In contrast, mRNA expression of AFP did not change at the highest concentration of 
DEN. Nope expression patterns changed as cells differentiated into more defined hepatic cell types. Furthermore, Nopepositive cells also expressed E-cadherin, and merged images revealed that Nope was expressed only in E-cadherin-positive cells, indicating that all Nope-positive cells were of epithelial origin.

Clinically established screening methods for HCC such as ultrasound and elevated serum levels of AFP have slightly improved the accuracy of prognosis, but their sensitivity and specificity are still limited, particularly at the early stages of tumor development $(7,33,34)$. Moreover, clinically established tumor markers fail to detect up to one-third of HCC cases. In addition to high sensitivity and reliable detection of early stages in carcinogenesis, the expression of ideal tumor markers should be limited in normal tissue and correlate with disease stage. Apart from AFP, other biomarkers, including AFP-L3, Golgi protein 73 , and des- $\gamma$-carboxy prothrombin (DCP), have been identified, and some show promising results for both screening and the evaluation of prognosis (35-38). However, none fulfills all the aforementioned criteria, although combinations of several markers might increase diagnostic power and prognostic reliability. Therefore, the identification of additional, more reliable tumor markers is particularly relevant $(16,17)$.

The expression of other biomarkers in HCC samples has been shown to generally be lower than that of Nope, and does not reach statistical significance in comparison to AFP. Notably, a previous study suggested that the expression levels of AFP and GPC-3 are regulated by similar mechanisms (39).

DEN-treated cells were stained positive for Nope, and Nope was specifically detected at the cell membrane during the early stages of hepatocarcinogenesis by confocal microscopy. Nopepositive cells co-expressed epithelial-specific E-cadherin. Nope was significantly overexpressed in DEN-treated hepatic lineage cells compared to untreated cells.

In conclusion, we identified that Nope may have prognostic significance during early hepatocarcinogenesis. These results indicate that Nope is a sensitive and specific marker for the early stages of hepatocarcinogenesis, and may have a superior detection rate compared to AFP. Nope is a novel oncofetal surface marker for preneoplastic stages in which the commonly used marker AFP is not yet overexpressed. This study contributes to cancer research by examining Nope expression during the early stages of hepatocarcinogenesis. Further investigations will concentrate on the functional and prognostic significance of Nope following treatment with other carcinogens.

\section{Acknowledgements}

We would like to thank Dr. Hwan-Goo Kang (Toxicology and Residue Chemistry Division, Animal, Plant and Fisheries Quarantine and Inspection Agency, MIFAFF) for his help and comments. This study was supported by the Basic Science Research Program through the National Research Foundation of Korea (NRF) funded by the Ministry of Education, Science and Technology (2012-0002704).

\section{References}

1. Greenhough S, Medine CN and Hay DC: Pluripotent stem cell derived hepatocyte like cells and their potential in toxicity screening. Toxicology 278: 250-255, 2010.
2. Okura H, Komoda H, Saga A, et al: Properties of hepatocyte-like cell clusters from human adipose tissue-derived mesenchymal stem cells. Tissue Engineering Part C Methods 16: 761-770, 2010.

3. Hamazaki T, Iiboshi Y, Oka M, et al: Hepatic maturation in differentiating embryonic stem cells in vitro. FEBS Lett 497: 15-19, 2001.

4. Touboul T, Hannan NR, Corbineau S, et al: Generation of functional hepatocytes from human embryonic stem cells under chemically defined conditions that recapitulate liver development. Hepatology 51: 1754-1765, 2010.

5. Rambhatla L, Chiu C-P, Kundu P, Peng Y and Carpenter MK: Generation of hepatocyte-like cells from human embryonic stem cells. Cell Transplant 12: 1-11, 2003.

6. Abelev GI, Perova SD, Khramkova NI, Postnikova ZA and Irlin IS: Production of embryonal alpha-globulin by transplantable mouse hepatomas. Transplantation 1: 174-180, 1963.

7. Bruix J and Sherman M; Practice Guidelines Committee, American Association for the Study of Liver Diseases: Management of hepatocellular carcinoma. Hepatology 42: 1208-1236, 2005.

8. Visvader JE and Lindeman GJ: Cancer stem cells in solid tumours: accumulating evidence and unresolved questions. Nat Rev Cancer 8: 755-768, 2008

9. Schievenbusch S, Schrammel T, Goeser T and Nierhoff D: Neighbor of Punc E11 in the Mdr2 $2^{-/}$mouse model: Novel marker of stem/progenitor cells in regenerating adult liver. In: Hepatology. Wiley-Blackwell, MA, pp 962A-962A, 2011.

10. Schievenbusch S, Sauer E, Curth HM, et al: Neighbor of Punc E 11: expression pattern of the new hepatic stem/progenitor cell marker during murine liver development. Stem Cells 21: 2656-2666, 2012.

11. Salbaum JM and Kappen C: Cloning and expression of nope, a new mouse gene of the immunoglobulin superfamily related to guidance receptors. Genomics 64: 15-23, 2000.

12. Marquardt JU, Quasdorff M, Varnholt H, et al: Neighbor of Punc E11, a novel oncofetal marker for hepatocellular carcinoma. Int J Cancer 128: 2353-2363, 2011.

13. He G, Dhar D, Nakagawa H, et al: Identification of liver cancer progenitors whose malignant progression depends on autocrine IL-6 signaling. Cell 155: 384-396, 2013.

14. Sato Y, Nakata K, Kato Y, et al: Early recognition of hepatocellular carcinoma based on altered profiles of alpha-fetoprotein. N Engl J Med 328: 1802-1806, 1993.

15. Sell S and Dunsford H: Evidence for the stem cell origin of hepatocellular carcinoma and cholangiocarcinoma. Am J Pathol 134: 1347, 1989.

16. Gebo KA, Chander G, Jenckes MW, et al: Screening tests for hepatocellular carcinoma in patients with chronic hepatitis $\mathrm{C}$ : a systematic review. Hepatology 36: S84-S92, 2002.

17. Gupta S, Bent S and Kohlwes J: Test characteristics of $\alpha$-fetoprotein for detecting hepatocellular carcinoma in patients with hepatitis C: A systematic review and critical analysis. Ann Intern Med 139: 46-50, 2003.

18. Marrero JA and Feng Z: Alpha-fetoprotein in early hepatocellular carcinoma. Gastroenterology 138: 400-401, 2010.

19. Forner A, Reig M and Bruix J: $\alpha$-fetoprotein for hepatocellular carcinoma diagnosis: the demise of a brilliant star. Gastroenterology 137: 26-29, 2009.

20. Marrero JA, Feng Z, Wang Y, et al: Alpha-fetoprotein, des-gamma carboxyprothrombin, and lectin-bound alpha-fetoprotein in early hepatocellular carcinoma. Gastroenterology 137: 110-118, 2009.

21. Kubota H and Reid LM: Clonogenic hepatoblasts, common precursors for hepatocytic and biliary lineages, are lacking classical major histocompatibility complex class I antigen. Proc Natl Acad Sci USA 97: 12132-12137, 2000.

22. Suzuki A,Zheng YW, Kondo R, et al: Flow-cytometric separation and enrichment of hepatic progenitor cells in the developing mouse liver. Hepatology 32: 1230-1239, 2000.

23. Watanabe T, Nakagawa K, Ohata S, et al: SEK1/MKK4-mediated SAPK/JNK signaling participates in embryonic hepatoblast proliferation via a pathway different from NF-kappaB-induced anti-apoptosis. Dev Biol 250: 332-347, 2002.

24. Tanimizu N, Nishikawa M, Saito H, Tsujimura T and Miyajima A: Isolation of hepatoblasts based on the expression of Dlk/Pref-1. J Cell Sci 116: 1775-1786, 2003.

25. Nierhoff D, Ogawa A, Oertel M, Chen YQ and Shafritz DA: Purification and characterization of mouse fetal liver epithelial cells with high in vivo repopulation capacity. Hepatology 42 : 130-139, 2005. 
26. Nitou M, Sugiyama Y, Ishikawa K and Shiojiri N: Purification of fetal mouse hepatoblasts by magnetic beads coated with monoclonal anti-e-cadherin antibodies and their in vitro culture. Exp Cell Res 279: 330-343, 2002.

27. Rabes H: Development and growth of early preneoplastic lesions induced in the liver by chemical carcinogens. J Cancer Res Clin Oncol 106: 85-92, 1983.

28. Kang JS, Kang HG, Park YI, et al: Expression of epithelial cell adhesion molecule and proliferating cell nuclear antigen in diethylnitrosamine induced hepatocarcinogenesis in mice. Exp Ther Med 5: 138-142, 2013

29. Kang JS: Expression of epithelial cell adhesion molecule in early phase of hepatocarcinogenesis of mice treated with diethylnitrosamine. J Biomed Res 13: 243-247, 2012.

30. Fausto $\mathrm{N}$ and Campbell JS: Mouse models of hepatocellular carcinoma. In: Seminars in Liver Disease. Thieme Medical Publishers, pp087-098, 2010. doi: 10.1055/s-002-20812.

31. Kang JS, Wanibuchi H, Morimura K, Gonzalez FJ and Fukushima S: Role of CYP2E1 in diethylnitrosamine-induced hepatocarcinogenesis in vivo. Cancer Res 67: 11141-11146, 2007

32. Bannasch P: Sequential cellular changes during chemical carcinogenesis. J Cancer Res Clin Oncol 108: 11-22, 1984.

33. Zhang BH, Yang BH and Tang ZY: Randomized controlled trial of screening for hepatocellular carcinoma. J Cancer Res Clin Oncol 130: 417-422, 2004.
34. Song BC, Chung YH, Kim JA, et al: Transforming growth factor- $\beta 1$ as a useful serologic marker of small hepatocellular carcinoma. Cancer 94: 175-180, 2002.

35. Li D, Mallory T and Satomura S: AFP-L3: a new generation of tumor marker for hepatocellular carcinoma. Clin Chim Acta 313 $15-19,2001$

36. Marrero JA, Su GL, Wei W, et al: Des-gamma carboxyprothrombin can differentiate hepatocellular carcinoma from nonmalignant chronic liver disease in American patients. Hepatology 37: 1114-1121, 2003.

37. Capurro M, Wanless IR, Sherman M, et al: Glypican-3: a novel serum and histochemical marker for hepatocellular carcinoma. Gastroenterology 125: 89-97, 2003.

38. Marrero JA, Romano PR, Nikolaeva O, et al: GP73, a resident Golgi glycoprotein, is a novel serum marker for hepatocellular carcinoma. J Hepatol 43: 1007-1012, 2005.

39. Morford LA, Davis C, Jin L, Dobierzewska A, Peterson ML and Spear BT: The oncofetal gene glypican 3 is regulated in the postnatal liver by zinc fingers and homeoboxes 2 and in the regenerating liver by alpha-fetoprotein regulator 2 . Hepatology 46 : 1541-1547, 2007. 